\title{
Coexisting single-particle solutions in low-density symmetric nuclear matter
}

\author{
H. F. Arellano* \\ Department of Physics-FCFM, University of Chile, Santiago, Chile. \\ E-mail: arellanoddfi.uchile.cl
}

\section{J.-P. Delaroche}

CEA, DAM, DIF, F-91297 Arpajon, France.

E-mail: jean-paul.delaroche@cea.fr

\author{
Arnau Rios \\ Department of Physics, Faculty of Engineering and Physical Sciences, University of Surrey, \\ Guildford, Surrey GU2 7XH, United Kingdom \\ E-mail:a.rios@surrey.ac.uk
}

The only two-nucleon bound state system occurring in free space is the deuteron, constituted by a proton-neutron pair. Although the neutron-neutron interaction is attractive, its strength is not deep enough to allow for a bound state in the form of a dineutron. However, this picture changes drastically when the interacting neutrons are immersed in nuclear matter. In this contribution we address dinucleon properties as implied by the Brueckner-Hartree-Fock approximation for infinite symmetric nuclear matter at zero $(T=0)$ and finite temperature. Special emphasis is given to dinucleon formation in the search of self-consistent single-particle fields, leading to novel features for low-density nuclear matter, i.e. mass densities of the order of $10^{11-12} \mathrm{~g} \mathrm{~cm}^{-3}$. Searches have been carried out at Fermi momenta in the range $0<k_{F} \leq 1.75 \mathrm{fm}^{-1}$ using the Argonne $v_{18}$ bare nucleon-nucleon potential. At $T=0$, two distinct solutions meeting self-consistency are found with overlapping domains in the interval $0.130 \mathrm{fm}^{-1} \leq k_{F} \leq 0.285 \mathrm{fm}^{-1}$. Effective masses as high as three times the nucleon mass are found in the coexistence domain, in resemblance to Heavy Fermions in strongly correlated systems. Self-consistent solutions for the single-particle fields at finite temperature are also investigated and preliminary results shall be presented.

$X$ Latin American Symposium on Nuclear Physics and Applications (X LASNPA),

1-6 December 2013

Montevideo, Uruguay

\footnotetext{
* Speaker.
} 


\section{Introduction}

The Brueckner, Bethe and Goldstone (BBG) theory for interacting nucleons has played an important role in the quest for a unified description of nuclear many-body phenomena based on the bare interaction between nucleons [1,2]. At negative energies in the Brueckner-Hartree-Fock (BHF) approximation it has been extensively applied to the study of saturation properties of nuclear matter, including the equation of state over the whole range of isospin-asymmetry. At positive energies, as in the case of nucleon scattering from nuclei, the $g$ matrix in the BHF approximation has been used to model density-dependent effective interactions between the projectile and target nucleons $[3,4]$.

Nuclear matter properties have been investigated within various frameworks such as microscopic quantum statistical approach and generalized relativistic mean field models [5], self-consistent Green's function theory [6,7], quasiparticle gas models for the study of light nuclei in supernova envelopes [8], among many others. However, there is no retrievable reference known to these authors where dinucleon bound state correlations are explicitly treated, in conjunction with the self-consistent search of single-particle (sp) fields within the BHF approximation, particularly at very low densities. In this work we address this issue by providing an explicit account for dinucleon bound states in the ${ }^{1} \mathrm{~S}_{0}$ and ${ }^{3} \mathrm{~S}_{1}-{ }^{3} \mathrm{D}_{1}$ channels, expressed as isolated poles on the real axis below the continuum threshold, while seeking self-consistency within the continuous choice.

\section{Framework}

In BBG theory for symmetric nuclear matter the $g$ matrix depends on the density of the medium, characterized by the Fermi momentum $k_{F}$, and starting energy $\omega$ [2]. To lowest order in the BHF approximation, when only two-body correlations are taken into account, the $g$ matrix satisfies

$$
g(\omega)=v+v \frac{Q}{\omega+i \eta-\hat{h}_{1}-\hat{h}_{2}} g(\omega)
$$

with $v$ the bare interaction between nucleons, $\hat{h}_{1}\left(\hat{h}_{2}\right)$ the sp energy of nucleon $1(2)$, and $Q$ the Pauli blocking operator. The solution to this equation enables the evaluation of the mass operator

$$
M(k ; E)=\sum_{|\mathbf{p}| \leq k_{F}}\left\langle\frac{1}{2}(\mathbf{k}-\mathbf{p})\left|g_{\mathbf{K}}\left(E+e_{p}\right)\right| \frac{1}{2}(\mathbf{k}-\mathbf{p})\right\rangle,
$$

where $\mathbf{K}$ is the total momentum of the interacting pair, $\mathbf{K}=\mathbf{k}+\mathbf{p}$, and

$$
e_{p}=\frac{p^{2}}{2 m}+U(p)
$$

the sp energy defined in terms of an auxiliary field $U$. The nucleon mass $m$ is taken as the average of proton and neutron masses. In the BHF approximation the sp potential is given by the on-shell mass operator,

$$
U(k)=\mathfrak{R} e M\left(k ; e_{k}\right),
$$

a self-consistency requirement which can only be achieved iteratively. In the continuous choice this condition is imposed to all momenta $k$ [2]. 
A standard procedure to solve the non-linear system of Eqs. (2.1-2.4) is by iterative feedbacking, where the auxiliary field $U$ is initially unknown. Cycles begin with a tentative guess for $U(k)$, which allows a solution for $g$ from Eq. (2.1) for the specified values of $\omega$ and $K$. The series of cycles can be started with $U_{0}(k)=0$, to obtain all $g$-matrix elements needed for the evaluation of $M$ in Eq. (2.2). The real part of $M$ defines a new sp field, $U_{1}$, which becomes the guess for a new cycle. The procedure is iterated until differences between consecutive solutions for $U$ meet some convergence criterion. Although this self-consistent scheme works well at normal densities, convergence may become extremelly difficult -if not unfeasible- as $k_{F}$ diminishes below $\sim 0.8 \mathrm{fm}^{-1}$. In this low-density regime, instabilities develop in the evaluation of the on-shell mass operator. We have noted that self-consistency at low densities can be reached by diminishing the number of mesh points for the Fermi-motion integral in Eq. (2.2). With this numerical compromise it is possible to avoid sudden fluctuations of the integrands, but the actual convergence of the integrals becomes dubious.

Aiming to obtain reliable self-consistent solutions for the sp mean fields over a wide density regime as well as accounting explicitly for dinucleon bound states, a refinement of numerical techniques becomes crucial to control instabilities during the iterative process. The evaluation of the sp fields, $U(k)=\Re e M\left(k, e_{k}\right)$, involves the summation of on-shell $g$-matrix elements while keeping the momentum of one of the particles below the Fermi surface. Explicitly,

$$
M\left(k, e_{k}\right)=\sum_{\alpha} n_{\alpha} \int_{0}^{k_{F}} p^{2} d p \int_{-1}^{1} d u g_{\mathbf{k}+\mathbf{p}}^{\alpha}\left(\frac{|\mathbf{k}-\mathbf{p}|}{2}, \frac{|\mathbf{k}-\mathbf{p}|}{2} ; \omega\right) .
$$

Here $u=\hat{\mathbf{k}} \cdot \hat{\mathbf{p}}$, the energy $\omega$ is evaluated on-shell $\left(\omega=e_{k}+e_{p}\right), \alpha$ denotes spin, isospin and angular momentum states, and $n_{\alpha}$ accounts for their degeneracy and for geometric factors.

For most $N N$ states the above integral involves well behaved integrands. However, extra measures becomes much needed for the ${ }^{1} \mathrm{~S}_{0}$ and ${ }^{3} \mathrm{~S}_{1}-{ }^{3} \mathrm{D}_{1}$ states. This is because during the evaluation of the momentum integral in Eq. (2.5), exceedingly large $g$-matrix elements stemming from $N N$ bound states in these $\mathrm{S}$ and D channels do appear. Such bound states occur when

$$
\operatorname{det}\left[1-v_{\alpha} \Lambda_{K}(\omega)\right]=0
$$

where $\Lambda_{K}(\omega)$ denotes the particle-particle propagator in Eq. (2.1) and $v_{\alpha}$ denotes the bare potential in a particular $N N$ channel. Any matrix element with starting energy $\omega$ and total momentum $K$ near such bound states will lead to large contributions. In actual calculations these singularities can be controlled with the regularization

$$
g(\omega) \rightarrow g(\omega) \times \frac{(\Delta \omega)^{2}}{(\Delta \omega)^{2}+\eta^{2}},
$$

where $\Delta \omega=\omega-\omega_{K}^{*}$. $\omega_{K}^{*}$ is the starting energy corresponding to a bound state of total momentum $K$, and $\eta$ is reasonably small real parameter. We have used $\eta=0.1 \mathrm{MeV}$, less than $5 \%$ of the deuteron binding energy in free space. The Fermi-motion integral is performed following an adaptive trapezoidal quadrature to ensure convergence. 


\section{Results}

Self-consistent solutions for $U(k)$ were obtained in the range $0<k_{F} \leq 1.75 \mathrm{fm}^{-1}$ using the Argonne $v_{18}$ bare internucleon potential [9]. We have included all $N N$ partial waves up to $J=7$, the total angular momentum. This criterion was maintained at all densities in order to cross-check our findings near the saturation point and to rule out any subtle variation of the calculated observables as caused by a discrete (sudden) change of in the number of partial waves. With these specifications, each cycle at which $U$ is calculated may involve of the order of half a million matrix inversion operations.

Different strategies were followed in searches for the self-consistent solutions for the cases $0<k_{F}<0.35 \mathrm{fm}^{-1}$ and $k_{F} \geq 0.35 \mathrm{fm}^{-1}$. In the second case, we calculated the solutions at Fermi momenta $k_{F}=0.35(0.05) 1.75 \mathrm{fm}^{-1}$, obtaining unique stable self-consistent solutions. In contrast, searches in the range $0<k_{F}<0.35 \mathrm{fm}^{-1}$, required a specific strategy due to the fact that two different self-consistent solutions are found for the same value of $k_{F}$. These two solutions, meeting self-consistency criteria, shall be referred to as coexisting solutions. To proceed systematically, a first class of solutions was obtained with ascending $k_{F}$, in steps of $\Delta k_{F}=0.01 \mathrm{fm}^{-1}$. At each $k_{F}$, the self-consistent loop starts with an $U(k)$ borrowed from the converged solution at the previous $k_{F}$. This way, starting at $k_{F}=0.01 \mathrm{fm}^{-1}$, we obtain a class of self-consistent solutions characterized by a monotonically increasing binding energy per nucleon, $B / A$, as a function of $k_{F}$. This class of solutions is denoted as $U_{I}=\left\{U_{k_{F}} \mid 0 \leq k_{F} \leq k_{\beta}\right\}$, with $k_{\beta}$ the maximum (critical) Fermi momentum at which self-consistency is achievable in ascending order. To resolve $k_{\beta}$, self-consistent solutions were explored with increasing $k_{F}$ in steps $\Delta k_{F}$ as small as $0.001 \mathrm{fm}^{-1}$, obtaining $k_{\beta}=0.285 \mathrm{fm}^{-1}$. Beyond this point the iteration loops indefinitely, with fluctuations in $U(k)$ unable to settle below $0.04 \mathrm{MeV}$, the maximum fluctuation required for convergence. A more refined characterization of the solutions near and beyond $k_{\beta}$ may require a more specific approach. A similar procedure was adopted for decreasing $k_{F}$, starting from the converged solution at $k_{F}=0.35 \mathrm{fm}^{-1}$ and going down in steps of $0.01 \mathrm{fm}^{-1}$. As in the previous case, the continuity of $B / A$ is monitored and we seek the minimum critical value of $k_{F}$, denoted by $k_{\alpha}$, at which self-consistency is reachable with decreasing $k_{F}$. This second class of solutions is denoted $U_{I I}=\left\{U_{k_{F}} \mid k_{F} \geq k_{\alpha}\right\}$. For these solutions we have found $k_{\alpha}=0.130 \mathrm{fm}^{-1}$, with a resolution of $0.01 \mathrm{fm}^{-1}$.

In Fig. 1 we show surface plots for the self-consistent fields $U(k)$ for solutions I and II in the range $0.05 \mathrm{fm}^{-1} \leq k_{F} \leq 0.35 \mathrm{fm}^{-1}$. These solutions define distinct surfaces at densities corresponding to $k_{\alpha} \leq k_{F} \leq k_{\beta}$. The upper sheet, representing $U_{I}$, features a moderate repulsion at $k=0$, with a shallow ditch in the vicinity $k \sim 4 k_{F} / 3$. On the other hand the sheet for $U_{I I}$ starts with negative mean fields at $k=0$, showing a moderate decrease up to $k \sim k_{F}$ and then followed by a steep increase to reach $U(k) \rightarrow 0$. In both cases the slope of the self-consistent fields become negative at the Fermi surface, a feature that causes nucleon effective masses to be greater than the bare nucleon mass. Here we evaluate

$$
\frac{m^{*}}{m}=\left[1+\frac{m}{k} \frac{\partial U(k)}{\partial k}\right]^{-1}
$$

at $k=k_{F}$.

In Fig. 2 we plot the ratio $m^{*} / m$ as function of $k_{F}$ for the two solutions. Dark and light diamonds denote $U_{I}$ and $U_{I I}$, respectively. We note that in the coexistence range the unbound 


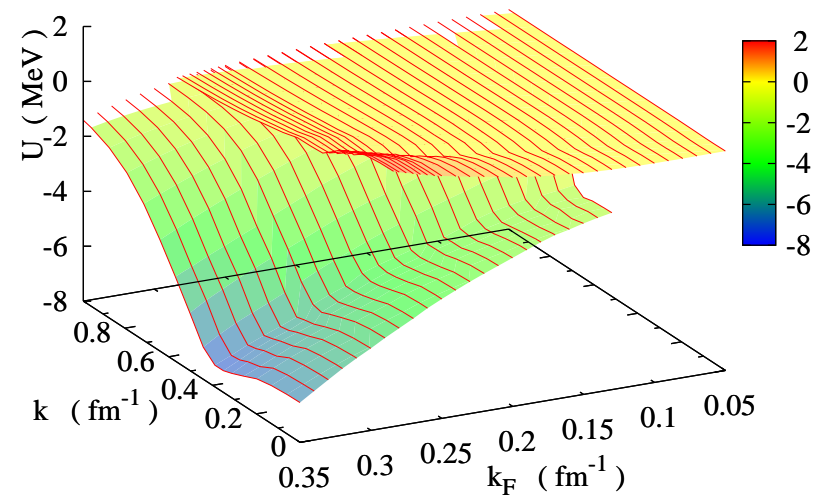

Figure 1: Self-consistent solutions at zero temperature for $U(k)$ as function of of $k$ and $k_{F}$. The upper sheet represents $U_{I}$ and the lower one corresponds to $U_{I I}$.

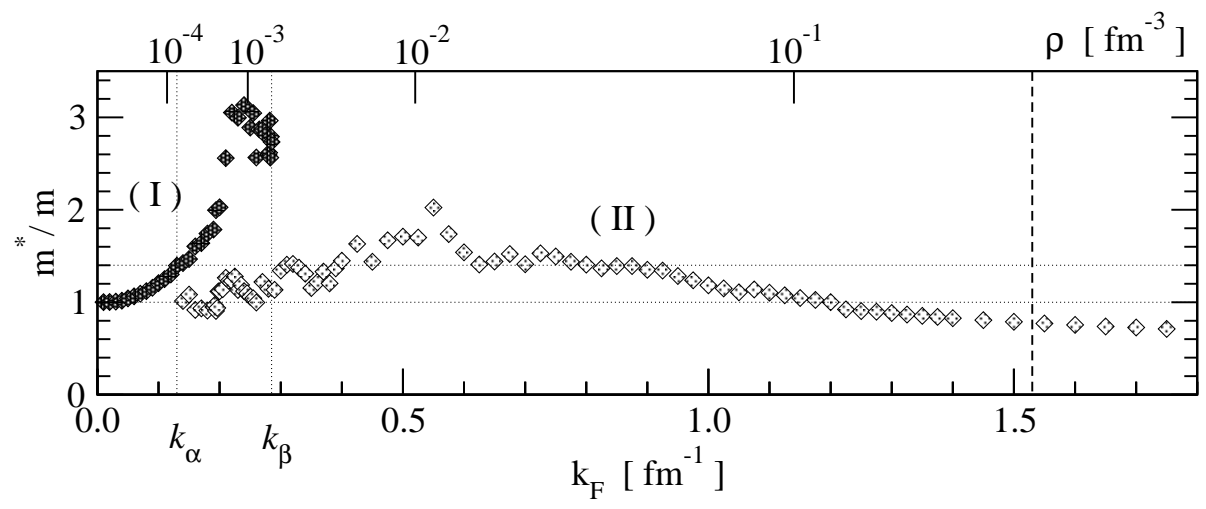

Figure 2: Effective mass $m^{*} / m$ as function of $k_{F}$ for solutions I and II.

solutions I yield effective masses as high as 3, consistent with the structure of the solutions for $k \sim k_{F}$, in resemblance to Heavy Fermions in strongly correlated systems [10].

In Fig. 3 we plot the binding energy per nucleon for symmetric nuclear matter, $B_{I} / A$ and $B_{I I} / A$, as obtained from solutions $U_{I}$ and $U_{I I}$, respectively. Here each small dot denotes an actual calculation, while the continuous curves represent suitable parametrizations. We note that the two solutions exhibit distinct behaviors as functions of $k_{F}$ in their respective domains, without intercepting each other in the range $\left[k_{\alpha}, k_{\beta}\right]$. While solution I resembles a correlated Fermi gas (FG) in a metastable state, solution II represents a condensed medium featuring a minimum at the point of nuclear saturation. From the inset for low densities we also note that solution I departs from an uncorrelated FG above $k_{\alpha}$, the Fermi momentum where solution II begins. This departure features a moderate repulsiveness relative to a free FG.

We have also investigated the occurence of dinucleon bound states at finite temperature. In this case we follow a naive extension of the BHF approach, where the density of states at a given 


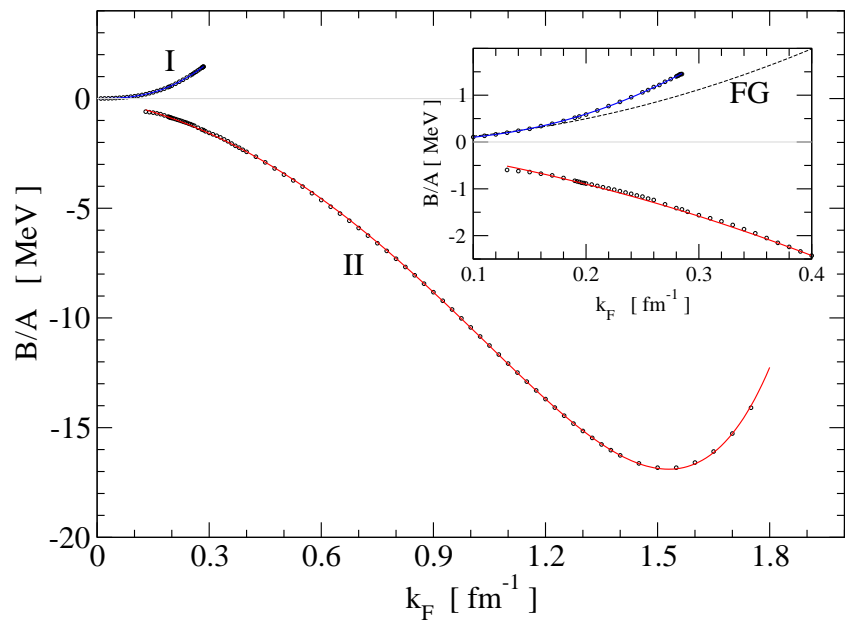

Figure 3: Calculated binding energy per nucleon $B / A$ for symmetric nuclear matter as a function of the Fermi momentum $k_{F}$. The solid blue (red) curve corresponds to solution I (II) based on power fits. Small circles denote results from actual self-consistent runs. The inset shows a close up for the coexisting solutions together with the Fermi gas result.

momentum $k$ is given by

$$
n(k)=\Theta\left(e_{F}-e_{k}\right) \rightarrow \frac{1}{1+e^{\left(e_{k}-\mu\right) / T}},
$$

with $e_{F}$ the Fermi energy and $\mu$ the chemical potential [11]. The latter is found self-consistently by imposing

$$
\rho=2 \int \frac{d^{3} k}{(2 \pi)^{3}} n(k),
$$

to match the density of nucleons. Additionally, the Pauli blocking operator in Eq. (2.1) is modified accordingly

$$
Q=\Theta\left(k_{1}-k_{F}\right) \Theta\left(k_{2}-k_{F}\right) \rightarrow\left[1-n\left(k_{1}\right)\right]\left[1-n\left(k_{2}\right)\right] .
$$

All other numerical procedures used in this case are the same as in the case of zero temperature. Results for the self-consistent solutions are shown in Fig. 4. The application here is for $k T=$ $0.2 \mathrm{MeV}$, displaying also coexisting solutions for $k_{F}$ above $0.2 \mathrm{fm}^{-1}$. Eventually, finite temperature calculations will provide us with a critical temperature, above which coexistence breaks down. Our current estimates suggest this is below $1 \mathrm{MeV}$. We note that the calculations we report in this work are computationally very intensive, and the strategy of reaching convergence to generate solutions in ascending/descending $k_{F}$ prevents us from resorting to parallel runs.

\section{Concluding remarks}

We have investigated dinucleon formation in homogeneous symmetric nuclear matter at $T=0$ and $T>0$. In this work it has been crucial to treat the sp solutions $U(k)$ without resorting to the effective-mass approximation, a consideration which has led us to identify coexisting selfconsistent sp fields in the range $0.13 \leq k_{F} \leq 0.285 \mathrm{fm}^{-1}$. Work in progress also suggests coexisting 


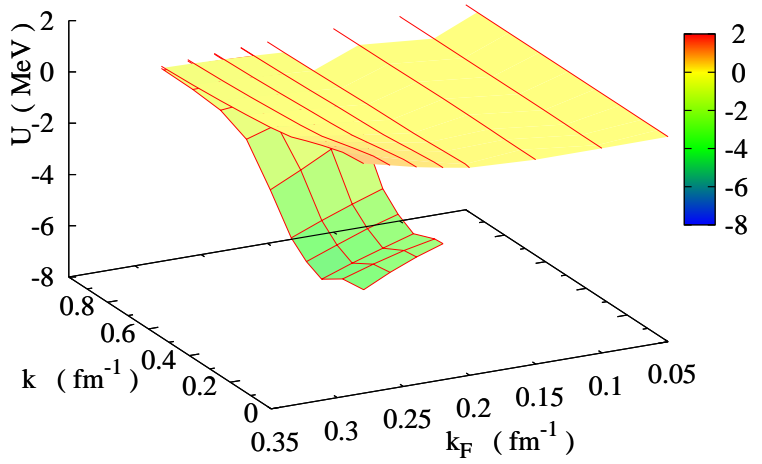

Figure 4: Self-consistent sp fields as function of $k$ and $k_{F}$ at finite temperature $(k T=0.2 \mathrm{MeV})$.

solutions at finite temperature, but further research is needed to pin down the temperature at which coexistence disappears. Although not discussed above, studies at zero temperature for the particleparticle wavefunctions yield bound states with sizes much greater than the internucleon distance, stressing the importance of long-range correlations. This fact has been inferred from the evaluation of mean radii, which can reach values as large as $\sim 100 \mathrm{fm}$. A more detailed report of these findings shall be published elsewhere.

\section{Acknowledgments}

Work supported in part by FONDECYT under grant No 1120396. A. Rios work is supported by STFC under grants ST/I005528/1 and ST/J000051 and Mobility Award from Santander Universities. H.F.A. acknowledges funding and the generous hospitality of colleagues at CEA/DAM/DIF, Bruyères-le-Châtel, France, where part of the research was done.

\section{References}

[1] W. H. Dickhoff and D. Van Neck, Many-Body Theory Exposed! (World Scientific, Singapore, 2008).

[2] M. Baldo (Ed.), in Nuclear Methods and the Nuclear Equation of State, International Review of Nuclear Physics, Vol. 8 (World Scientific, Singapore, 1999).

[3] H. F. Arellano and E. Bauge, Phys. Rev. C 84, 034606 (2011).

[4] K. Amos, P. J. Dortmans, H. V. von Geramb, S. Karataglidis, and J. Raynal, in Advances in Nuclear Physics, Vol. 25, edited by J. W. Negele and E. Vogt (Plenum, New York, 2000).

[5] S. Typel, G. Röpke, T. Klähn, D. Blaschke, and H. H. Wolter, Phys. Rev. C 81, 015803 (2010).

[6] B. E. Vonderfecht, C. C. Gearhart, W. H. Dickhoff, A. Polls and A. Ramos, Phys. Lett. B 253, 1 (1991).

[7] A. Ramos, A. Polls and W. H. Dickhoff, Nucl. Phys. A 503, 1 (1989).

[8] S. Heckel, P. P. Schneider, and A. Sedrakian, Phys. Rev. C 80, 015805 (2009). 
[9] R. B. Wiringa, V. G. J. Stoks, and R. Schiavilla, Phys. Rev. C 51, 38 (1995).

[10] H. v. Lohneysen, A. Rosch, M. Vojta, and P. Wolfle, Rev. Mod. Phys. 79, 1015 (2007).

[11] M. Baldo and L. Ferreira, Phys. Rev. C 59, 682 (1999). 